\title{
Portfolio Optimization in Discontinuous Markets under Incomplete Information
}

\author{
Giorgia Callegaro, Giovanni B. Di Masi, \\ and Wolfgang J. Runggaldier
}
Dipartimento di Matematica Pura ed Applicata, Universitá di Padova, Via Trieste, 63, I-35121 Padova, Italy e-mails : giorgia.callegaro@tin.it, dimasi@math.unipd.it, runggal@math.unipd.it

\begin{abstract}
We consider the problem of maximization of expected utility from terminal wealth for $\log$ and power utility functions in a market model that leads to purely discontinuous processes. We study this problem as a stochastic control problem both under complete as well as incomplete information. Our contribution consists in showing that the optimal strategy can be obtained by solving a system of equations that in some cases is linear and that a certainty equivalence property holds not only for log-utility but also for a power utility function. For the case of a power utility under incomplete information we also present an independent direct approach based on a Zakai-type equation.
\end{abstract}

Mathematics Subject Classification : Primary 93E20; Secondary 91B28

Keywords : Portfolio optimization, Stochastic control, Discontinuous Markets, Incomplete information.

\section{Introduction}

Portfolio optimization problems are important in Finance and they belong to the general class of optimal stochastic control problems. The majority of these problems concern diffusion-type models. Here we consider a market model that leads to a purely discontinuous state process. Such models may be of general interest in stochastic control. In a financial context they may arise e.g. when asset prices are observed over small time scales, in particular in the case of high frequency data. In such a context the price trajectories are typically piecewise constant and jump only at random discrete points in time in reaction to trading or significant new information (for a more detailed discussion on this see e.g. [5]). While in such a context one observes frequent jumps, discontinuous models with less frequent jumps may arise 
whenever small changes in prices are neglected and only major price movements are registered as a jump. This may in particular be the case when one considers the value of an index rather than prices of individual assets. Such values evolve along trajectories presenting small fluctuations for most of the time with major movements only now and then. Introducing some fixed bands in the trajectory space one may thus consider a jump to have occurred whenever the price/value exits a given band. We shall show that for such purely discontinuous processes the solution to the portfolio optimization problem can be obtained rather easily. It follows that, by neglecting smaller price/value movements, one has a considerable advantage for what concerns the computation of an optimal investment strategy.

In our portfolio optimization problem we limit ourselves to maximization of expected utility from terminal wealth for two common utility functions : log-utility and power utility in the risk averse case.

As for general stochastic control problems, also for portfolio optimization not all quantities of relevance in a given model may be known or observable. This corresponds then to the case of incomplete information and here an interesting property is that of certainty equivalence $(\mathrm{CE})$ : the solution of the incomplete information problem is obtained from that of the complete information counterpart by replacing the unknown quantities by their current conditional expectations. We shall show that, in our context, such a property holds not only for log-utility, but also for power utility functions.

In Section 2 we describe more precisely the model and the problem; the possibly unknown quantities are the intensities of the driving Poisson processes. In Section 3 we study the log-utility case using both a direct method that turns out to be equivalent to Dynamic programming (DP) as well as a method based on convex duality. We show that an optimal strategy can be obtained by solving a system of equations that in particular cases becomes linear. In Section 4 we treat the case of a power utility and show that also in this case an optimal strategy can be obtained by solving a system of equations and that a CE property holds, which is obtained by applying a particular measure transformation. In subsection 4.2 we present an independent direct approach for the case of partial information and this approach is inspired by [10] and [11].

\section{Market model and problem definition}

We consider a market with a non-risky asset having, without loss of generality, a price given by $B_{t} \equiv 1$ and $d$ risky assets, the prices of which evolve on a filtered probability space $\left(\Omega, \mathcal{F}, \mathcal{F}_{t}, \mathbb{P}\right)$ according to pure-jump processes, namely

$$
\mathrm{d} S_{t}^{i}=S_{t^{-}}^{i}\left[\sum_{j=1}^{m}\left(e^{a_{i j}}-1\right) \mathrm{d} N_{t}^{j}\right] \quad i=1, \ldots, d
$$

where $a_{i j}$ are deterministic constants and $N_{t}^{j}, j=1, \ldots, m$, with $m>d$ (incomplete market) are point processes without common jumps having intensities $\lambda_{t}^{j}$. The latter can be characterized (see [1, Ch. II, T4]) by the fact that for all nonnegative 
predictable processes $\left\{C_{t}\right\}$ and all $t \geq 0$ we have

$$
E\left\{\int_{0}^{t} C_{s} \mathrm{~d} N_{s}^{j}\right\}=E\left\{\int_{0}^{t} C_{s} \lambda_{s}^{j} \mathrm{~d} s\right\} ; \quad j=1, \cdots, m
$$

It is well known that the solution to the stochastic equation (2.1) is given by

$$
S_{t}^{i}=S_{0}^{i} \exp \left[\sum_{j=1}^{m} a^{i j} N_{t}^{j}\right] \quad \text { for } i=1, \cdots, d .
$$

We consider both the case when the values of the intensities can be explicitly observed (complete information case) as well as the case when only the prices $\left\{S_{t}^{i}\right\}$ are observed (incomplete information). In the latter case the values of the intensities have to be estimated on the basis of the observed prices so that a filtering problem arises. For the explicit solution of the filtering problem we shall need to model the time evolution of the intensity process $\lambda_{t}=\left[\lambda_{t}^{1}, \ldots, \lambda_{t}^{m}\right]^{\prime}$ and for this we shall assume that it forms an homogeneous Markov process with generator $\mathcal{L}_{t}$. In particular, and in order to get more explicit expressions (see the second part of Section 3.3 and Section 4.2), we shall assume that the homogeneous Markov process $\lambda_{t}$ takes a finite number $K$ of possible values so that the generator $\mathcal{L}_{t}$ is then given by the transition intensity matrix $Q$.

Remark 1. In the Introduction we had mentioned that our simple purely discontinuous model can, in a financial context, also be motivated by neglecting smaller fluctuations in prices/values and registering only major changes as a jump. These jumps occur when the price/value process exits a given band (the fact that these bands are a priori given justifies the assumption that $a^{i j}$ are deterministic constants also in such a context). Now exit times are predictable while the jump times of a Poisson process are totally inaccessible. This apparent discrepancy between our formal model and the above justification in a financial context can be overcome on the basis of recent results concerning crossing times (see e.g. [6], [7]), from which it follows that also crossing times become totally inaccessible if they are observed with delay or in noise.

In the given market we are interested in a portfolio optimization problem, for simplicity without consumption. To this effect recall that, for a self-financing strategy characterized by the predictable fractions $h_{t}^{i}$ of wealth invested in $S_{t}^{i}, i=1, \ldots, d$, the portfolio value $V_{t}$ satisfies

$$
\frac{\mathrm{d} V_{t}}{V_{t^{-}}}=\sum_{i=1}^{d} h_{t}^{i} \frac{\mathrm{d} S_{t}^{i}}{S_{t^{-}}^{i}}=\sum_{i=1}^{d} h_{t}^{i} \cdot \sum_{j=1}^{m}\left(e^{a_{i j}}-1\right) \mathrm{d} N_{t}^{j}
$$

We shall assume that, in order to be admissible, an investment strategy $h_{t}=$ $\left(h_{t}^{1}, \cdots, h_{t}^{d}\right)$ satisfies

$$
\sum_{i=1}^{d} h_{t}^{i}\left(e^{a_{i j}}-1\right) \geq-1, \quad \forall j=1, \cdots, m, \quad \text { a.s. }
$$


One then has that, at a given final time $T$,

$$
V_{T}=V_{0} \cdot \prod_{j=1}^{m} \exp \left[\int_{0}^{T} \log \left(1+\sum_{i=1}^{d} h_{t}^{i}\left(e^{a_{i j}}-1\right)\right) \mathrm{d} N_{t}^{j}\right]>0
$$

Our optimization problem concerns both the logarithmic as well as the power utility functions in the risk-averse case. In other words, we shall consider the problem of maximizing, with respect to $h_{t}:=\left(h_{t}^{1}, \ldots, h_{t}^{d}\right)$, the expected value of

$$
\log V_{T}=\log V_{0}+\sum_{j=1}^{m} \int_{0}^{T} \log \left(1+\sum_{i=1}^{d} h_{t}^{i}\left(e^{a_{i j}}-1\right)\right) \mathrm{d} N_{t}^{j}
$$

and

$$
V_{T}^{\mu}=V_{0}^{\mu} \cdot \prod_{j=1}^{m} \exp \left[\int_{0}^{T} \log \left(1+\sum_{i=1}^{d} h_{t}^{i}\left(e^{a_{i j}}-1\right)\right)^{\mu} \mathrm{d} N_{t}^{j}\right]
$$

with $0<\mu<1$ respectively.

The portfolio optimization problems will be studied in the next sections both in the case of complete information and in the case of incomplete (partial) information.

The case of logarithmic utility (2.6) will be studied in the next Section 3, while the power utility (2.7) will be examined in Section 4.

It is known that, for the portfolio optimization problem to make sense, the market model should not allow for the possibility of arbitrage. Equivalently, there should exist an equivalent martingale measure (EMM), namely a probability measure $\mathbb{Q}$ under which the discounted asset prices are martingales. It will be seen later in the paper that, in our context, the optimal value is finite for both utility functions and so there is no possibility of arbitrage. Here, in the last part of this section we conclude by describing the possible martingale measures as this will be used below, in particular for the martingale approach.

To this end it is known (see [1]) that in the case of Poisson processes an absolutely continuous measure change implies a change of the intensities $\lambda_{t}^{j}, j=1, \ldots, m$ into new values $\lambda_{t}^{j} \psi_{t}^{j}, j=1, \ldots, m$, where $\psi_{t}^{j} \geq 0$ are $\mathcal{F}_{t}$-predicable and satisfy, for all $t \in[0, T]$

$$
\int_{0}^{t} \psi_{s}^{j} \lambda_{s}^{j} \mathrm{~d} s<\infty, \quad \mathbb{P}-\text { a.s. }
$$

The corresponding measure transformation is described by a Radon-Nikodym derivative of the form

$$
\frac{\mathrm{d} \mathbb{Q}}{\mathrm{d} \mathbb{P}}{ }_{\mid \mathcal{F}_{t}}=Z_{t}=\exp \left\{\sum_{j=1}^{m} \int_{0}^{t}\left(1-\psi_{s}^{j}\right) \lambda_{s}^{j} \mathrm{~d} s+\sum_{j=1}^{m} \int_{0}^{t} \log \psi_{s}^{j} \mathrm{~d} N_{s}^{j}\right\}
$$

Furthermore, to guarantee that (2.9) represents a Radon-Nikodym derivative of two probability measures, one should have

$$
E^{\mathbb{P}}\left\{Z_{T}\right\}=1
$$


and a sufficient condition for this can be found in [1, Ch. VIII, T11].

Under the new measure $\mathbb{Q}$ the processes

$$
\mathrm{d} M_{t}^{j}=\mathrm{d} N_{t}^{j}-\lambda_{t}^{j} \psi_{t}^{j} \mathrm{~d} t \quad j=1, \ldots, m
$$

are martingales and the price processes admit the representation

$$
\mathrm{d} S_{t}^{i}=S_{t^{-}}^{i}\left\{\sum_{j=1}^{m}\left(e^{a_{i j}}-1\right) \mathrm{d} M_{t}^{j}+\sum_{j=1}^{m}\left(e^{a_{i j}}-1\right) \lambda_{t}^{j} \psi_{t}^{j} \mathrm{~d} t\right\} \quad i=1, \ldots, d
$$

It is then clear that the prices, which coincide in our case with the discounted prices, are themselves martingales under $\mathbb{Q}$ if and only if the $\psi_{t}^{j}$ 's satisfy for all $i=1, \ldots, d$ the relations

$$
\sum_{j=1}^{m}\left(e^{a_{i j}}-1\right) \lambda_{t}^{j} \psi_{t}^{j}=0
$$

with $m>d$. We assume that this system admits solutions and that they are parametrized by $k \geq m-d$ parameters. Therefore, we can think of the EMM's as being parametrized by the vector process

$$
\nu_{t}=\left(\nu_{t}^{1}, \ldots, \nu_{t}^{k}\right) \in \mathcal{N}
$$

where $\mathcal{N}$ is the set of processes such that the resulting $\psi$ 's are nonnegative and such that (2.8), (2.10) and (2.13) are satisfied, so that $\mathcal{N}$ actually parametrizes the set of EMM.

\section{Logarithmic utility function}

In this section we shall study the portfolio optimization problem for a logarithmic utility function, namely the problem of determining a predictable portfolio $h_{t}=$ $\left(h_{t}^{1}, \ldots, h_{t}^{d}\right)$ which attains the optimal value

$$
\sup _{h} E\left\{\log V_{T}^{h}\right\}=\log V_{0}+\sup _{h} \sum_{j=1}^{m} E\left\{\int_{0}^{T} \log \left(1+\sum_{i=1}^{d} h_{t}^{i}\left(e^{a_{i j}}-1\right)\right) \mathrm{d} N_{t}^{j}\right\} .
$$

Assuming that, in addition to (2.4), the admissible strategies satisfy also

$$
\int_{0}^{T}\left[\log \left(1+\sum_{i=1}^{d} h_{t}^{i}\left(e^{a_{i j}}-1\right)\right)\right]^{-} \lambda_{t}^{j} \mathrm{~d} t<\infty, \quad \forall j=1, \ldots, m, \quad \text { a.s. }
$$

where $[Z]^{-}$denotes the negative part of $Z$, this optimal value can be rewritten as

$$
\sup _{h} E\left\{\log V_{T}^{h}\right\}=\log V_{0}+\sup _{h} \sum_{j=1}^{m} E\left\{\int_{0}^{T} \log \left(1+\sum_{i=1}^{d} h_{t}^{i}\left(e^{a_{i j}}-1\right)\right) \lambda_{t}^{j} \mathrm{~d} t\right\}
$$




\subsection{A direct approach}

If the intensities $\lambda_{t}$ can be directly observed, then for the optimal investment strategy it suffices to maximize for each $t \in[0, T]$ and each $\lambda_{t}$ (observed)

$$
C\left(h_{t}, \lambda_{t}\right):=\sum_{j=1}^{m} \log \left(1+\sum_{i=1}^{d} h_{t}^{i}\left(e^{a_{i j}}-1\right)\right) \lambda_{t}^{j}
$$

Due to the concavity of the function (3.4), by differentiating with respect to $h$, we immediately have the following

Proposition 2. A necessary and sufficient condition for a portfolio $h_{t}$ to be optimal in the optimization problem (3.3) is that it satisfies the following system of equations:

$$
\sum_{j=1}^{m} \frac{\lambda_{t}^{j}\left(e^{a_{i j}}-1\right)}{1+\sum_{i=1}^{d} h_{t}^{i}\left(e^{a_{i j}}-1\right)}=0 \quad i=1, \ldots, d
$$

with the constraint (3.2) as well as with $1+\sum_{i=1}^{d} h_{t}^{i}\left(e^{a_{i j}}-1\right)>0$.

Remark 3. Notice that in the special case when $m=2$ the system (3.5) becomes a linear system of $d$ equations in the $d$ unknowns $h_{t}^{j}(j=1, \ldots, d)$. For $m>2$ the system is nonlinear.

Remark 4 (Dynamic Programming approach). The same result can also be obtained via dynamic programming techniques under the assumption that $\lambda_{t}$ is a homogeneous Markov process with generator $\mathcal{L}_{t}$. In fact, defining the value function

$$
w(t, \lambda):=\sup _{h} E\left\{\int_{t}^{T} C\left(h_{s}, \lambda_{s}\right) d s \mid \lambda_{t}=\lambda\right\}
$$

with $C\left(h_{t}, \lambda_{t}\right)$ as given in (3.4), so that

$$
\sup _{h} E\left\{\log V_{T} \mid \lambda_{t}=\lambda, V_{t}=v\right\}=\log v+w(t, \lambda)
$$

the corresponding HJB equation is given by

$$
\frac{\partial}{\partial t} w(t, \lambda)+\mathcal{L}_{t} w(t, \lambda)+\sup _{h}[C(h, \lambda)]=0
$$

Since the investment strategy $h$ appears only in the last term, we obtain the same result given in Proposition 2.

\subsection{Martingale approach}

We shall now discuss an alternative approach that has some advantages with respect to the direct method just described and which will allow also the derivation of the optimal value. It is obtained by using techniques from the theory of convex duality 
(see e.g [9], see also the more recent developments as described e.g. in [15]). We shall briefly review a few results which are relevant in our context.

In order to apply convex duality techniques, the portfolio optimization problem will be studied in the framework of the so called "martingale approach", namely the constraint due to the initial wealth $V_{0}=v$ will be expressed as $E^{\mathbb{Q}}\left\{V_{T}^{h}\right\} \leq v$ for all EMM $\mathbb{Q}$ (recall that we had assumed $B_{t} \equiv 1$ ). According to the results in Section 2, the set of EMM can be parametrized by a multivariate process $\nu_{t}=\left(\nu_{t}^{1}, \ldots, \nu_{t}^{k}\right) \in \mathcal{N}$. Consequently, this set will be denoted by $\left\{Q^{\nu}, \nu \in \mathcal{N}\right\}$.

We can then reformulate as follows our problem that in the context of convex duality will be called primal problem, namely

$$
\left\{\begin{array}{l}
\max _{h} E\left\{u\left(V_{T}^{h}\right)\right\} \\
E^{\mathbb{Q}^{\nu}}\left[V_{T}^{h}\right] \leq v \forall \nu=\left(\nu_{t}\right) \in \mathcal{N}
\end{array}\right.
$$

Its solution can be obtained in two steps: in the first step one determines $V_{T}^{*}$ such that

$$
\left\{\begin{array}{l}
\max _{V_{T} \in \mathcal{V}_{v}} E\left\{u\left(V_{T}\right)\right\}=E\left\{u\left(V_{T}^{*}\right)\right\} \\
\mathcal{V}_{v}=\left\{V_{T}: E^{\mathbb{Q}^{\nu}}\left[V_{T}\right] \leq v \forall \nu=\left(\nu_{t}\right) \in \mathcal{N}\right\}
\end{array}\right.
$$

In the second step one then determines the optimal strategy $h^{*}$ such that

$$
V_{T}^{h^{*}}=V_{T}^{*} \quad \mathbb{P} \text {-a.s. }
$$

According to [9], for each initial wealth $v$ we can associate to the primal problem the dual functional

$$
\begin{aligned}
L(\nu, \gamma) & :=\sup _{V_{T}}\left[E\left\{u\left(V_{T}\right)\right\}-\gamma E^{\mathbb{Q}^{\nu}}\left\{V_{T}\right\}+\gamma v\right] \\
& =\sup _{V_{T}}\left[E\left\{u\left(V_{T}\right)-\gamma Z_{T}^{\nu} V_{T}\right\}\right]+\gamma v
\end{aligned}
$$

where $Z_{T}^{\nu}$ denotes the Radon-Nikodym derivative $\frac{d \mathbb{Q}^{\nu}}{d \mathbb{P}}$.

Since the maximization can be performed for each $\omega \in \Omega$, we have:

$$
L(\nu, \gamma)=\gamma v+E\left\{\tilde{u}\left(\gamma Z_{T}^{\nu}\right)\right\}
$$

where $\tilde{u}(y):=\sup _{x>0} u(x)-x y, y>0$ is the convex dual of $u(\cdot)$.

The associated dual problem is described by

$$
\left\{\begin{array}{l}
\min _{\nu, \gamma} L(\nu, \gamma) \\
\nu \in \mathcal{N}, \gamma>0
\end{array}\right.
$$

It is known [9, Theorem 1, Ch. 7.12$]$ that for each admissible solution $\bar{V}_{T}$ of the primal problem (3.9) and each admissible solution $(\bar{\nu}, \bar{\gamma})$ of the dual problem (3.12) we have

$$
E\left\{u\left(\bar{V}_{T}\right)\right\} \leq L(\bar{\nu}, \bar{\gamma})
$$

and for any triple $V_{T}^{*}, \nu^{*}, \gamma^{*}$ achieving equality in (3.13), $V_{T}^{*}$ is optimal for the primal problem and $\left(\nu^{*}, \gamma^{*}\right)$ is optimal for the dual.

Using the above arguments it is possible to derive the optimal strategy and the optimal value of the portfolio for a log-utility function. This is done in the following 
Proposition 5. In the case of a logarithmic utility function $u(x)=\log (x)$, if $\left(\nu^{*}, \gamma^{*}\right)$ is the optimal solution to the dual problem, then

$$
V_{T}^{*}=\frac{1}{\gamma^{*} Z_{T}^{\nu^{*}}}
$$

is the optimal solution to problem (3.9) and therefore the optimal strategy $h^{*}$ can be obtained via a martingale representation of

$$
M_{t}=E^{\mathbb{Q}^{*}}\left\{V_{T}^{*} \mid \mathcal{F}_{t}\right\}=E^{\mathbb{Q}^{*}}\left\{\frac{v}{Z_{T}^{\nu^{*}}} \mid \mathcal{F}_{t}\right\}=\frac{v}{Z_{t}^{\nu^{*}}}
$$

where $Q^{*}=Q^{\nu^{*}}$.

Proof. For $u(x)=\log (x)$ we have from (3.11) that the dual functional is given by

$$
L(\nu, \gamma)=\gamma v-1+E\left\{\log \frac{1}{\gamma Z_{T}^{\nu}}\right\}
$$

so that the minimum with respect to $\gamma$ is attained in

$$
\gamma^{*}=\frac{1}{v}
$$

Then (3.13) is satisfied as an equality for

$$
V_{T}^{*}=\frac{1}{\gamma^{*} Z_{T}^{\nu^{*}}}=\frac{v}{Z_{T}^{\nu^{*}}}
$$

It now remains to prove that (3.16) satisfies the constraint in (3.9) so that it provides the optimal solution to the primal problem. This follows from Theorem 2.2 in [16].

The explicit derivation of the optimal strategy via the martingale representation of (3.15) is based on standard arguments.

Remark 6. Notice that for $m=d+1$ the set of the EMM is parametrized by a scalar process $\nu_{t}$ which appears linearly in the solution of the system of equations (2.13) and consequently in the $\psi_{t}^{j}$ 's in (2.9). Using the expression of $Z_{t}$ thus obtained, it is easily seen that in this case the solution to the dual problem (3.12) requires the solution of a linear equation in the unknown $\nu_{t}$.

On the contrary, for $m>d+1$, one has to solve a system of nonlinear equations.

Remark 7. The approach to portfolio optimization based on convex duality leads thus to a linear system of equations for $m=d+1$ and in this situation it is more convenient than the direct approach (and the equivalent one based on dynamic programming) which leads to a linear situation only for $m=2$. 


\subsection{Partial observations and certainty equivalence}

For the case when the intensities $\lambda_{t}$ are not directly observed a certainty-equivalence property holds.

In fact, since in this case $h_{t}$ is measurable with respect to the filtration $\mathcal{F}_{t}^{S}=\mathcal{F}_{t}^{N}$ generated by the price process, equation (3.3) can be rewritten with $\hat{\lambda}_{t}^{j}=E\left\{\lambda_{t}^{j} \mid \mathcal{F}_{t}^{S}\right\}$ replacing $\lambda_{t}$.

As a consequence, the portfolio optimization problem can be solved using the formulas derived above with $\lambda_{t}^{j}$ replaced by $\hat{\lambda}_{t}^{j}$. This certainty equivalence property for $\log$ utilities can be shown to hold also for a rather general class of Levy-driven market models (see e.g. [12]).

The derivation of $\hat{\lambda}_{t}$ requires the solution of a filtering problem. For the case when $\lambda_{t}$ is a finite state Markov process with intensity matrix $Q$, its solution is studied in [1] and summarized next.

Denote by $\phi^{i}, i=1, \ldots, K$ the (vector) values assumed by the process $\lambda_{t}$, with $\phi^{i}=\left[\phi_{1}^{i}, \phi_{2}^{i}, \ldots, \phi_{m}^{i}\right]^{\prime}$ and by $\phi_{i}, i=1, \ldots, m$ the vector $\phi_{i}=\left[\phi_{i}^{1}, \ldots, \phi_{i}^{K}\right]^{\prime}$. Denote

$$
\hat{Z}_{t}^{i}:=\mathbb{P}\left(\lambda_{t}=\phi^{i} \mid \mathcal{F}_{t}^{N}\right) \quad i=1, \ldots, K
$$

the corresponding vector

$$
\hat{Z}_{t}=\left[\hat{Z}_{t}^{1}, \ldots, \hat{Z}_{t}^{K}\right]^{\prime}
$$

the a priori probabilities $p_{0}=\left[p_{0}^{1}, \ldots, p_{0}^{K}\right]^{\prime}$ with $p_{0}^{i}=\mathbb{P}\left(\lambda_{0}=\phi^{i}\right)$ and the matrix

$$
M_{i}=\left(\begin{array}{ccc}
\phi_{i}^{1} & & \\
& \ddots & \\
& & \phi_{i}^{K}
\end{array}\right)=\operatorname{diag}\left\{\phi_{i}\right\}
$$

We then have the recursive formula

$$
\hat{Z}_{t}=p_{0}+\int_{0}^{t} Q^{\prime} \hat{Z}_{s} \mathrm{~d} s+\sum_{i=1}^{m} \int_{0}^{t}\left(-\hat{Z}_{s}+\frac{M_{i} \hat{Z}_{s^{-}}}{\phi_{i}^{\prime} \hat{Z}_{s^{-}}}\right)\left(\mathrm{d} N_{s}^{i}-\phi_{i}^{\prime} \hat{Z}_{s}\right) \mathrm{d} s
$$

Finally, defining the matrix $\Phi=\left\{\phi_{j}^{i}\right\}_{i=1, \ldots, K ; j=1, \ldots, m}$ we have that the (vector) estimator of $\lambda_{t}$ is given by

$$
\hat{\lambda}_{t}=\hat{Z}_{t}^{\prime} \Phi
$$

\section{Power-utility function}

We shall study here the case of the power-utility function (2.7), so that the portfolio optimization problem consists in determining a predictable portfolio $h_{t}=\left(h_{t}^{1}, \ldots, h_{t}^{d}\right)$ which attains the optimal value

$$
\sup _{h} E\left\{V_{T}^{\mu}\right\}=\sup _{h} V_{0}^{\mu} \cdot E\left\{\prod_{j=1}^{m} \exp \left[\int_{0}^{T} \log \left(1+\sum_{i=1}^{d} h_{t}^{i}\left(e^{a_{i j}}-1\right)\right)^{\mu} \mathrm{d} N_{t}^{j}\right]\right\}
$$




\subsection{A direct approach}

Here we try to parallel as much as possible the approach of subsection 3.1 for the logarithmic case. We first obtain an expression, equivalent to (4.1), with an integral with respect to the time variable. By analogy to an approach in [11] (see also [10]) we rewrite (4.1) for the case when $\lambda_{t}$ is known as

$$
\begin{aligned}
\sup _{h} E\left\{V_{T}^{\mu}\right\}= & \sup _{h} V_{0}^{\mu} \cdot E\left\{\prod _ { j = 1 } ^ { m } \operatorname { e x p } \left[\int_{0}^{T} \log \left(1+\sum_{i=1}^{d} h_{t}^{i}\left(e^{a_{i j}}-1\right)\right)^{\mu} \mathrm{d} N_{t}^{j}\right.\right. \\
& +\int_{0}^{T}\left[1-\left(1+\sum_{i=1}^{d} h_{t}^{i}\left(e^{a_{i j}}-1\right)\right)^{\mu}\right] \lambda_{t}^{j} \mathrm{~d} t \\
& \left.\left.-\int_{0}^{T}\left[1-\left(1+\sum_{i=1}^{d} h_{t}^{i}\left(e^{a_{i j}}-1\right)\right)^{\mu}\right] \lambda_{t}^{j} \mathrm{~d} t\right]\right\}
\end{aligned}
$$

and therefore, if

$$
\begin{gathered}
E\left\{\prod _ { j = 1 } ^ { m } \operatorname { e x p } \left[\int_{0}^{T} \log \left(1+\sum_{i=1}^{d} h_{t}^{i}\left(e^{a_{i j}}-1\right)\right)^{\mu} \mathrm{d} N_{t}^{j}\right.\right. \\
\left.\left.+\int_{0}^{T}\left[1-\left(1+\sum_{i=1}^{d} h_{t}^{i}\left(e^{a_{i j}}-1\right)\right)^{\mu}\right] \lambda_{t}^{j} \mathrm{~d} t\right]\right\}=1
\end{gathered}
$$

so that the argument of the expectation in (4.3) represents the Radon-Nikodym derivative of a transformation of the probability $\mathbb{P}$ into a probability $\mathbb{P}^{h}$ (see $[1, \mathrm{Ch}$. VI, T3]), the optimization problem becomes

$$
\sup _{h} E\left\{V_{T}^{\mu}\right\}=\sup _{h} V_{0}^{\mu} \cdot E^{h}\left\{\prod_{j=1}^{m} \exp \left[\int_{0}^{T}\left[\left(1+\sum_{i=1}^{d} h_{t}^{i}\left(e^{a_{i j}}-1\right)\right)^{\mu}-1\right] \lambda_{t}^{j} \mathrm{~d} t\right]\right\}
$$

and notice that, under the measure $\mathbb{P}^{h}$, the intensity of $N_{t}^{j}$ is $\lambda_{t}^{j}\left(1+\sum_{i=1}^{d} h_{t}^{i}\left(e^{a_{i j}}-1\right)\right)^{\mu}$ while the law of $\lambda_{t}$ remains the same.

Remark 8. Using [1, Ch. VIII, T11] it is clear that a sufficient condition for (4.3) is given by

$$
\sum_{i=0}^{d}\left(h_{t}^{i}\right)^{-} \leq c
$$

where $c$ is a positive constant and where we have denoted by $h_{t}^{0}$ the fraction of wealth invested in the non-risky asset $B_{t}$.

As a consequence

$$
\sum_{i=0}^{d}\left(h_{t}^{i}\right)^{+} \leq 1+c
$$

Notice also that the above condition is implied by (2.4). 
The optimization problem (4.4) is of the form of a risk-sensitive control problem and so its solution could be obtained by using DP techniques. In our case we can however follow a direct approach analogously to what we did in subsection 3.1 for the logarithmic case. In fact, if the intensities $\lambda_{t}$ can be directly observed, in order to maximize (4.4) it suffices to maximize for each $t \in[0, T]$ and each $\lambda_{t}$

$$
\bar{C}\left(h_{t}, \lambda_{t}\right):=\sum_{j=1}^{m}\left[\left(1+\sum_{i=1}^{d} h_{t}^{i}\left(e^{a_{i j}}-1\right)\right)^{\mu}-1\right] \lambda_{t}^{j}
$$

which, for $\mu \in(0,1)$, is again a concave function of $h_{t}$. Differentiating then with respect to $h$ we immediately have the following result, analogous to that of Proposition 2 .

Proposition 9. A necessary and sufficient condition for a portfolio $h_{t}$ to be optimal in the optimization problem (4.4) is that it satisfies the following system of equations:

$$
\sum_{j=1}^{m} \frac{\lambda_{t}^{j}\left(e^{a_{i j}}-1\right)}{\left(1+\sum_{i=1}^{d} h_{t}^{i}\left(e^{a_{i j}}-1\right)\right)^{1-\mu}}=0, \quad i=1, \ldots, d
$$

with the constraint that $\sum_{i=1}^{d} h_{t}^{i}\left(e^{a_{i j}}-1\right) \geq-1$.

The maximization in (4.6) leads here always to a nonlinear system of equations.

Remark 10 (Partial observation and certainty equivalence). In the case when the intensities $\lambda_{t}$ are not directly observed a certainty equivalence property holds here as well.

In fact, in this case the investment strategy $h_{t}$ has to be adapted to the observation filtration $\mathcal{F}_{t}^{S}=\mathcal{F}_{t}^{N}$ and so it is possible to repeat the steps in (4.2) replacing $\lambda_{t}^{j}$ with $\hat{\lambda}_{t}^{j}, j=1, \ldots, m$ that, when $\lambda_{t}$ is finite state Markov, can be determined as described at the end of subsection 3.3 .

Since $\hat{\lambda}_{t}^{j}$ is the $\mathcal{F}_{t}^{S}$-intensity of the process $N_{t}$, the measure transformation techniques described above apply also in this case and the $\mathbb{P}^{h}$-intensities are here $\hat{\lambda}_{t}^{j}\left(1+\sum_{i=1}^{d} h_{t}^{i}\left(e^{a_{i j}}-1\right)\right)^{\mu}$.

Notice that, while the measure transformation does not change the law of $\lambda_{t}$, it affects however the dynamics of $\hat{\lambda}_{t}$, i.e. the filter dynamics described at the end of section 3.3 have to be considered under the measure $\mathbb{P}^{h}$. This leads to an added complication with respect to the case of a log-utility and so in the next section we present an alternative approach for the case of a power utility function under partial information.

Remark 11. For the case of the power-utility function the technique based on convex duality is less direct than in the case of the log-utility. This technique is presently under investigation and preliminary results can be found in [2]. 


\subsection{An alternative approach for the partial information case}

\subsubsection{Reformulation of the problem}

Inspired by [10] and [11] we shall reformulate our problem on the basis of a Zakai-type equation.

We assume here more specifically that $\lambda_{t}$ is finite-state Markov and that it is obtained as follows : let $X_{t}$ be an external factor process that is an independent finite state Markov process with $K$ states and transition intensity matrix $Q$. Let then

$$
\lambda_{t}=\left[\lambda_{t}^{1}, \cdots, \lambda_{t}^{m}\right]^{\prime} \quad \text { with } \quad \lambda_{t}^{j}=\lambda^{j}\left(X_{t}\right), j=1, \cdots, m
$$

Next perform an absolutely continuous change of measures from $\mathbb{P}$ to $\hat{\mathbb{P}}$ such that $\frac{d \hat{\mathbb{P}}}{d \mathbb{P}} \mid \mathcal{F}_{t}=L_{t}$ with

$$
L_{t}=\prod_{j=1}^{m} \exp \left[\int_{0}^{t}\left(\lambda^{j}\left(X_{s}\right)-1\right) d s-\int_{0}^{t} \log \lambda^{j}\left(X_{s-}\right) d N_{s}^{j}\right]
$$

Since $X_{t}$ is finite-state, there exists $\bar{\lambda}$ such that

$$
\lambda^{j}\left(X_{t}\right) \leq \bar{\lambda}, \quad j=1, \cdots, m
$$

namely the intensities are bounded and so the conditions are fulfilled for (4.7) to be a Radon-Nikodym derivative. Under $\hat{\mathbb{P}}$ the intensities become $\lambda^{j}\left(X_{t}\right) \equiv 1$ and so $X_{t}$ and $N_{t}^{j}$ are $\hat{\mathbb{P}}$-independent $\forall j=1, \cdots, m$.

With the use of the above measure transformation we obtain that

$$
\begin{gathered}
E\left\{V_{T}^{\mu}\right\}=V_{0}^{\mu} \hat{E}\left\{\prod _ { j = 1 } ^ { m } \operatorname { e x p } \left[\int _ { 0 } ^ { T } \left\{\log \left(1+\sum_{i=1}^{d} h_{t}^{i}\left(e^{a_{i j}}-1\right)\right)^{\mu}\right.\right.\right. \\
\left.\left.\left.+\log \lambda^{j}\left(X_{t-}\right)\right\} d N_{t}^{j}-\int_{0}^{T}\left(\lambda^{j}\left(X_{t}\right)-1\right) d t\right]\right\} \\
=V_{0}^{\mu} \hat{E}\left\{\exp \left[\int_{0}^{T} \sum_{j=1}^{m} \log \Gamma^{j}\left(h_{t}, X_{t-}\right) d N_{t}^{j}-\int_{0}^{T} \sum_{j=1}^{m}\left(\lambda^{j}\left(X_{t}\right)-1\right) d t\right]\right\}
\end{gathered}
$$

having put

$$
\Gamma^{j}\left(h_{t}, X_{t}\right):=\lambda^{j}\left(X_{t}\right)\left(1+\sum_{i=1}^{d} h_{t}^{i}\left(e^{a_{i j}}-1\right)\right)^{\mu}
$$

Notice that, on the basis of the two inequalities in Remark 8, by the admissibility condition (2.4) for the investment strategy we have

$$
\begin{aligned}
& \left|\sum_{i=1}^{d} h_{t}^{i}\left(e^{a_{i j}}-1\right)\right| \leq \max _{i, j}\left|e^{a_{i j}}-1\right| \sum_{i=1}^{d}\left|h_{t}^{i}\right| \\
& =\max _{i, j}\left|e^{a_{i j}}-1\right| \sum_{i=1}^{d}\left(\left(h_{t}^{i}\right)^{+}+\left(h_{t}^{i}\right)^{-}\right) \leq(1+2 c) \max _{i, j}\left|e^{a_{i j}}-1\right|
\end{aligned}
$$

Taking also (4.8) into account it then follows that

$$
0<\Gamma^{j}\left(h_{t}, X_{t}\right) \leq \bar{\lambda}\left(1+(1+2 c) \max _{i, j}\left|e^{a_{i j}}-1\right|\right):=\bar{\Gamma}
$$


Motivated by the right hand side for $E\left\{V_{T}^{\mu}\right\}$ in (4.9), consider next as in [11] the process

$$
H_{t}:=\exp \left[\int_{0}^{t} \sum_{j=1}^{m} \log \Gamma^{j}\left(h_{s}, X_{s-}\right) d N_{s}^{j}-\int_{0}^{t} \sum_{j=1}^{m}\left(\lambda^{j}\left(X_{s}\right)-1\right) d s\right]
$$

and put

$$
q_{t}(k)=\hat{E}\left\{1_{\left\{X_{t}=k\right\}} H_{t} \mid \mathcal{F}_{t}^{S}\right\}, \quad k=1, \cdots, K
$$

From the bounds in (4.8) and (4.12) and taking into account the moment generating function of a Poisson random variable as well as the fact that, under $\hat{\mathbb{P}}$, the intensities are $\lambda^{j}\left(X_{t}\right) \equiv 1$, one obtains the following bounds for $\hat{E}\left\{H_{t}\right\}$ and $q_{t}(k),(k=1, \cdots, K)$

$$
\left\{\begin{array}{l}
0<\hat{E}\left\{H_{t}\right\} \leq e^{m T \bar{\Gamma}} e^{m T}:=\bar{q} \\
0<q_{t}(k)<\bar{q}, \quad k=1, \cdots, K
\end{array}\right.
$$

Furthermore, from (4.9) one then obtains

$$
\begin{aligned}
& E\left\{V_{T}^{\mu}\right\}=V_{0}^{\mu} \hat{E}\left\{H_{T}\right\}=V_{0}^{\mu} \hat{E}\left\{\hat{E}\left\{H_{T} \mid \mathcal{F}_{T}^{S}\right\}\right\} \\
& =V_{0}^{\mu} \sum_{k=1}^{K} \hat{E}\left\{\hat{E}\left\{1_{\left\{X_{T}=k\right\}} H_{T} \mid \mathcal{F}_{T}^{S}\right\}\right\}=V_{0}^{\mu} \hat{E}\left\{\sum_{k=1}^{K} q_{T}(k)\right\} \leq V_{0}^{\mu} K \bar{q}
\end{aligned}
$$

Proceeding by complete analogy to [11], using Ito's formula on $H_{t}$ and properties of finite-state Markov chains as well as the fact that the law of $X_{t}$ is the same under $\mathbb{P}$ and $\hat{\mathbb{P}}$, one obtains for the processes $q_{t}(k)$ as defined in $(4.14)$ the following dynamics

$$
\begin{aligned}
d q_{t}(k) & =\left(Q^{\prime} q_{t}\right)(k) d t+q_{t}(k) \sum_{j=1}^{m}\left(1-\lambda^{j}(k)\right) d t \\
& +q_{t-}(k) \sum_{j=1}^{m}\left(\Gamma^{j}\left(h_{t}, k\right)-1\right) d N_{t}^{j} ; \quad k=1, \cdots, K
\end{aligned}
$$

It furthermore follows that, defining $\pi_{t}(k):=\mathbb{P}\left\{X_{t}=k \mid \mathcal{F}_{t}^{S}\right\}$, one obtains $\pi_{t}(k)=$ $\frac{q_{t}(k)}{\sum_{j=1}^{K} q_{t}(j)}$, so that $q_{t}(k)$ has the interpretation of an unnormalized conditional probability.

Our portfolio problem for a power utility function and under incomplete information about the intensities of the driving Poisson processes can then be synthesized as (putting $\left.q_{t}=\left[q_{t}(1), \cdots, q_{t}(K)\right]^{\prime}\right)$

$$
\left\{\begin{array}{l}
\max _{h_{a d m}} V_{0}^{\mu} \hat{E}\left\{\sum_{k=1}^{K} q_{T}(k)\right\} \\
d q_{t}=\left[Q^{\prime}+\sum_{j=1}^{m}\left[I-\operatorname{diag}\left(\lambda^{j}(k)\right)\right]\right] q_{t} d t+\left[\sum_{j=1}^{m}\left(\operatorname{diag}\left(\Gamma^{j}\left(h_{t}, k\right)\right)-I\right) q_{t-} d N_{t}^{j}\right]
\end{array}\right.
$$


where $\operatorname{diag}(z(k))$ is used to denote the diagonal matrix with entries $z(1), \cdots, z(K)$, $h_{a d m}$ denote the strategies satisfying the admissibility condition (2.4) and the dynamics of $q_{t}$ are under the measure $\hat{\mathbb{P}}$ for which the intensities are all $\lambda^{j}\left(X_{t}\right) \equiv 1$.

\subsubsection{Solution approach}

Our reformulated problem in (4.18) is of the type of a piecewise deterministic control problem. We could thus try to adapt to our situation the more general approaches for the solution of such problems as described e.g. in [3], [4] or, more recently, in [13]. It turns out, however, that for our particular situation we can rather straightforwardly extend an approach followed in [8] for the solution of the hedging problem in a purely discontinuous market model corresponding to (2.1).

To simplify the expressions we first introduce some shorthand notations. The dynamics in (4.18) imply that, at the generic jump time $\tau^{j}$ of the $j$-th driving Poisson process $N_{t}^{j}$ one has

$$
q_{\tau^{j}}=\operatorname{diag}\left(\Gamma^{j}\left(h_{\tau^{j}}, k\right)\right) q_{\left(\tau^{j}\right)^{-}}
$$

while, between two generic jump times of the multivariate jump process $N_{t}=$ $\left(N_{t}^{1}, \cdots, N_{t}^{m}\right)$, i.e. for $t \in[\tau, \tau+1)$ one has the deterministic evolution

$$
d q_{t}=\left[Q^{\prime}+m I-\operatorname{diag}\left(\sum_{j=1}^{m} \lambda^{j}(k)\right)\right] q_{t} d t:=\Lambda q_{t} d t
$$

so that, always for $t \in[\tau, \tau+1)$,

$$
q_{t}=\exp [\Lambda(t-\tau)] \cdot q_{\tau}
$$

Let $\tau_{n}$ be the time of the $n$-th jump of the multivariate jump process $N_{t}=$ $\left(N_{t}^{1}, \cdots, N_{t}^{m}\right.$ ) before $T$ (if $\tau_{n}>T$ put $\tau_{n}=T$ ). By analogy to Lemma 2.2 in [8] and recalling that, under $\hat{\mathbb{P}}$, for all intensities one has $\lambda^{j}\left(X_{t}\right) \equiv 1$, we can state the following

Lemma 12. Given any function $f(q)$ of the process $q_{t}$, one has

$$
\begin{aligned}
\hat{E}\left\{f\left(q_{\tau_{n+1}}\right) \mid q_{\tau_{n}}=q\right\} & =\int_{\tau_{n}}^{T}\left[\sum_{j=1}^{m} f\left(\operatorname{diag}\left(\Gamma^{j}\left(h_{t}, k\right)\right) e^{\Lambda\left(t-\tau_{n}\right)} q\right)\right] e^{-m\left(t-\tau_{n}\right)} d t \\
& +f\left(e^{\Lambda\left(T-\tau_{n}\right)} q\right) e^{-m\left(T-\tau_{n}\right)}
\end{aligned}
$$

Proof : Notice first that

$$
\begin{aligned}
\hat{E} & \left\{f\left(q_{\tau_{n+1}}\right) \mid q_{\tau_{n}}=q\right\} \\
& =\hat{E}\left\{f\left(q_{\tau_{n+1}}\right), \tau_{n+1} \leq T \mid q_{\tau_{n}}=q\right\}+\hat{E}\left\{f\left(q_{\tau_{n+1}}\right), \tau_{n+1}>T \mid q_{\tau_{n}}=q\right\}
\end{aligned}
$$


By (4.21) it immediately follows that

$$
\hat{E}\left\{f\left(q_{\tau_{n+1}}\right), \tau_{n+1}>T \mid q_{\tau_{n}}=q\right\}=f\left(e^{\Lambda\left(T-\tau_{n}\right)} q\right) e^{-m\left(T-\tau_{n}\right)}
$$

Coming to the first term on the right in (4.23) and denoting by $\tau_{n+1}^{j}$ the first jump time of the $j$-th driving Poisson process $N_{t}^{j}$ after $\tau_{n}$, we have (see (4.19))

$$
\begin{aligned}
\hat{E} & \left\{f\left(q_{\tau_{n+1}}\right), \tau_{n+1} \leq T \mid q_{\tau_{n}}=q\right\} \\
& =\sum_{j=1}^{m} \hat{E}\left\{f\left(\operatorname{diag}\left(\Gamma^{j}\left(h_{\tau_{n+1}^{j}}, k\right)\right) q_{\left(\tau_{n+1}^{j}\right)^{-}}\right), \tau_{n+1}^{j}<\min _{i \neq j} \tau_{n+1}^{i}, \tau_{n+1}^{j} \leq T \mid q_{\tau_{n}}=q\right\} \\
& =\sum_{j=1}^{m} \hat{E}\left\{f\left(\operatorname{diag}\left(\Gamma^{j}\left(h_{\tau_{n+1}^{j}}, k\right)\right) q_{\left(\tau_{n+1}^{j}\right)^{-}}\right)\right. \\
& =\sum_{j=1}^{m} \int_{\tau_{n}}^{T} f\left(\operatorname{diag}\left(\Gamma^{j}\left(h_{t}, k\right)\right) q_{t^{-}}\right) \hat{\mathbb{P}}\left\{t<\min _{i \neq j} \tau_{n+1}^{i} \mid q_{\tau_{n}}=q\right\} \hat{\mathbb{P}}\left\{\tau_{n+1}^{j} \in d t \mid q_{\tau_{n}}=q\right\} \\
& \left.=\sum_{j=1}^{m} \int_{\tau_{n}}^{T} f\left(\operatorname{diag}\left(\Gamma^{j}\left(h_{t}, k\right)\right) \tau_{n+1}^{j} \leq T \mid \tau_{n+1}^{j}\right\} \mid q_{\tau_{n}}=q\right\} \\
& =\sum_{j=1}^{m} \int_{\tau_{n}}^{T} f\left(\operatorname{diag}\left(\Gamma^{j}\left(h_{t}, k\right)\right) q_{t^{-}}\right) e^{-(m-1)\left(t-\tau_{n}\right)} e^{-\left(t-\tau_{n}\right)} d t \\
& =\sum_{j=1}^{m} \int_{\tau_{n}}^{T} f\left(\operatorname{diag}\left(\Gamma^{j}\left(h_{t}, k\right)\right) e^{\Lambda\left(t-\tau_{n}\right)} d t e^{-m\left(t-\tau_{n}\right)} d t\right.
\end{aligned}
$$

The process $q_{t}$ with dynamics in (4.18) is a Markov process that, by (4.15), satisfies $0<q_{t}<\bar{q} \underline{1}$. For our problem we may thus consider as state space the following

$$
E=\left\{(q, t) \mid q \in \mathbb{R}^{K}, 0<q_{t}<\bar{q} \underline{1}, t \in[0, T]\right\}
$$

For $J: E \rightarrow \mathbb{R}^{+}$define the operator $\Psi$ mapping $J$ to $\Psi J: E \rightarrow \mathbb{R}^{+}$by

$$
\begin{aligned}
(\Psi J)(q, t) & =\int_{0}^{T-t} e^{-m s} \max _{h}\left[\sum_{j=1}^{m} J\left(\operatorname{diag}\left(\Gamma^{j}(h, k)\right) e^{\Lambda s} q, t+s\right)\right] d s \\
& +e^{-m(T-t)} \sum_{k=1}^{K}\left(e^{\Lambda(T-t)} q\right)^{k}
\end{aligned}
$$


where the last term on the right is motivated by the objective function in (4.18) $\left((V)^{k}\right.$ denotes the $k$-th component of the column vector $\left.V\right)$ and the max is over the values of $h=\left(h^{1}, \cdots, h^{d}\right)$ that satisfy the admissibility condition (2.4). As already taken into account in (4.11), by Remark 8 this latter condition implies that

$$
\sum_{i=1}^{d}\left|h_{t}^{i}\right| \leq \sum_{i=1}^{d}\left(h_{t}^{i}\right)^{+}+\sum_{i=1}^{d}\left(h_{t}^{i}\right)^{-} \leq 1+2 c
$$

so that the maximization is performed over a compact set. From here on we can now follow rather closely the development in [8]. In fact, denoting by $\mathcal{C}(E)$ the class of functions $J$ on $E$ such that $(q, t) \rightarrow J(q, t)$ is continuous, by an easily adapted proof one can next show

Lemma 13. The operator $\Psi: \mathcal{C}(E) \rightarrow \mathcal{C}(E)$ is a contraction with contraction constant $1-e^{-m T}$.

Next, given $n \in \mathbb{N}$, let

$$
J^{0}=0 \quad \text { and, for } j \leq n, \quad J^{j}=\Psi J^{j-1}
$$

and let $\left(h_{t}^{n}\right)_{t \in[0, T]}$ be the strategy induced by computing $J^{n}\left(q_{0}, 0\right)$ (for more details on this see Section 3.1 in [8]). Motivated always by the particular form of our objective function in (4.18), corresponding to (17) in [8] define

$$
J^{*, n}(q, t)=\max _{h_{a d m}} \hat{E}\left\{\sum_{k=1}^{K} q_{T}(k), \tau_{n}>T \mid q_{t}=q\right\}
$$

where, here, $\tau_{n}$ is the time of the $n$-th jump of the multivariate Poisson process $N_{t}$ after $t$. This definition implies that $J^{*, n}\left(q_{0}, 0\right)$ is the optimal value for the problem obtained from our original problem in (4.18) by replacing $\Omega$ with $\Omega \cap\left\{\tau_{n}>T\right\}$. By a proof that is completely analogous to that of Lemma 3.2 in [8] one then has

Lemma 14. The $n$-th iterate according to (4.29) corresponds to the optimal value of the original problem when at most $n$ jumps are taken into account, i.e.

$$
J^{n}(q, t)=J^{*, n}(q, t), \quad \forall(q, t)
$$

The following further lemma will allow us to prove the main Theorem 16 below.

Lemma 15. For $J^{*}(q, t)=\max _{h_{\text {adm }}} \hat{E}\left\{\sum_{k=1}^{K} q_{T}(k) \mid q_{t}=q\right\}$ we have the double inequality

$$
J^{n}(q, t) \leq J^{*}(q, t) \leq J^{n}(q, t)+K \bar{q} \hat{\mathbb{P}}\left\{\tau_{n} \leq T\right\}
$$

where $\bar{q}$ is the bound in (4.15) and, as in (4.30), $\tau_{n}$ denotes the $n$-th jump of the multivariate Poisson process $N_{t}=\left[N_{t}^{1}, \cdots, N_{t}^{m}\right]$ after $t$. 
Proof : For the first inequality we have

$$
\begin{aligned}
J^{*}(q, t) & =\max _{h_{\text {adm }}}\left[\hat{E}\left\{\sum_{k=1}^{K} q_{T}(k), \tau_{n}>T \mid q_{t}=q\right\}+\hat{E}\left\{\sum_{k=1}^{K} q_{T}(k), \tau_{n} \leq T \mid q_{t}=q\right\}\right] \\
& \geq \max _{h_{\text {adm }}} \hat{E}\left\{\sum_{k=1}^{K} q_{T}(k), \tau_{n}>T \mid q_{t}=q\right\}=J^{*, n}(q, t)=J^{n}(q, t)
\end{aligned}
$$

For the second inequality, using the bound on $q_{t}(k)$ in $(4.15)$, we have instead

$$
\begin{aligned}
J^{*}(q, t) & =\max _{h_{a d m}}\left[\hat{E}\left\{\sum_{k=1}^{K} q_{T}(k), \tau_{n}>T \mid q_{t}=q\right\}\right. \\
& \left.+\hat{E}\left\{\sum_{k=1}^{K} q_{T}(k) \mid q_{t}=q, \tau_{n} \leq T\right\} \hat{\mathbb{P}}\left\{\tau_{n} \leq T\right\}\right] \\
& \leq \max _{h_{a d m}} \hat{E}\left\{\sum_{k=1}^{K} q_{T}(k), \tau_{n}>T \mid q_{t}=q\right\}+K \bar{q} \hat{\mathbb{P}}\left\{\tau_{n} \leq T\right\} \\
& =J^{*, n}(q, t)+K \bar{q} \hat{\mathbb{P}}\left\{\tau_{n} \leq T\right\}=J^{n}(q, t)+K \bar{q} \hat{\mathbb{P}}\left\{\tau_{n} \leq T\right\}
\end{aligned}
$$

We can now state and prove the main

\section{Theorem 16.}

i) The optimal value function $J^{*}=J^{*}(q, 0)=\max _{h_{a d m}} \hat{E}\left\{\sum_{k=1}^{K} q_{T}(k) \mid q_{0}=q\right\}$ is the unique fixed point of the operator $\Psi$, i.e.

$$
J^{*}=\Psi J^{*}
$$

and we have

$$
\left\|J^{n}-J^{*}\right\| \leq e^{m T}\left(1-e^{-m T}\right)^{n}\left\|J^{1}\right\|
$$

ii) The following strategy $h^{*}$ is optimal : given a generic jump time $\tau_{n}$ of the multivariate Poisson process $N_{t}=\left(N_{t}^{1}, \cdots, N_{t}^{m}\right)$, for $t \in\left(\tau_{n}, \tau_{n+1}\right]$ and $q=q_{\tau_{n}}$ the value of $h_{t}^{*}$ is given by the solution, that exists, of the deterministic optimization problem embedded in (4.27), i.e.

$$
h_{t}^{*}=\operatorname{argmax}_{h}\left[\sum_{j=1}^{m} J^{*}\left(\operatorname{diag}\left(\Gamma^{j}(h, k)\right) e^{\Lambda\left(t-\tau_{n}\right)} q, t\right)\right]
$$

with $h$ satisfying (2.4). 
iii) Let $\tilde{J}^{n}=\tilde{J}^{n}(q, 0)$ be the value function of the original problem corresponding to the strategy $\left(h_{t}^{n}\right)$ induced by computing $J^{n}(q, 0)$. Then

$$
J^{n} \leq \tilde{J}^{n} \leq J^{n}+K \bar{q} \hat{\mathbb{P}}\left\{\tau_{n} \leq T\right\}
$$

Remark 17. Since $\lim _{n \rightarrow \infty} \hat{\mathbb{P}}\left\{\tau_{n} \leq T\right\}=0$, it follows from (4.33) and (4.35) that $\lim _{n \rightarrow \infty} \tilde{J}^{n}=J^{*}$, i.e. the strategy $\left(h_{t}^{n}\right)$, induced by computing the $n$-th iterate $J^{n}(q, 0)$ $\stackrel{n \rightarrow \infty}{\Psi}$, is, for $n$ sufficiently large, nearly optimal in the original problem.

Proof : The proof can, for most of its part, be adapted from that of the corresponding Theorem 3.3 in [8]. Item i) follows from Lemmas 13 and 15 by considerations analogous to those for the proof of item i) in Theorem 3.3 of [8]. Item ii) follows similarly by applying the Dynamic Programming Principle. The existence of a maximizer follows from the fact that, by the (uniform) convergence of $J^{n}$ to $J^{*}$, by Lemma 13 and (4.28) as well as the considerations following it, we have to maximize a continuous function over a compact set. Item iii) follows from considerations analogous to those in the proof of Lemma 15 taking into account the fact that $\tilde{J}^{n}(q, 0)=\max _{h} \hat{E}\left\{\sum_{k=1}^{K} q_{T}(k), \tau_{n}>T \mid q_{0}=q\right\}$.

To actually determine a nearly optimal investment strategy $h_{t}^{n}$, one has to be able to perform the iterations in (4.29), i.e. to compute the operator $\Psi$ in (4.27). For this purpose one has to discretize (quantize) the problem in the variables $(q, t)$ and then to interpolate it in the same variables. It is possible to control the error induced by this interpolation as well as by the stopping after the $n$-th iteration. All this can be done in complete analogy to [8] (for the numerical aspects see also [14]) and so we do not describe it here but refer to those articles.

\section{References}

[1] P. Bremaud, Point processes and queues, martingale dynamics. Springer-Verlag New York 1981.

[2] G. Callegaro, and T. Vargiolu, Optimal portfolio for HARA utility functions in a pure jump incomplete market. Preprint 2005.

[3] M. Davis, Markov Models and Optimization. Chapman \& Hall, London 1993.

[4] M.A.H. Dempster, Optimal Control of Piecewise Deterministic Markov Processes, Applied Stochastic Analysis (ed. M.H.A. Davis and R.J. Elliott), Stochastics Monogr., 5, Gordon and Breach, New York, 1991, 303-325.

[5] R. Frey, and W.J. Runggaldier, A Nonlinear Filtering Approach to Volatility Estimation with a View Towards High Frequency Data, International Journal of Theoretical and Applied Finance, 4, 2001, pp.199-210. 
[6] X. Guo, and Y. Zeng, Intensity process and compensator : A new filtration expansion approach and the Jeulin-Yor formula, Preprint, 2006.

[7] X. Guo, R.A. Jarrow, and Ch. Menn, A Generalized Lando's Formula : A Filtration Expansion Perspective, Preprint, 2006.

[8] M. Kirch and W.J. Runggaldier, Efficient hedging when asset prices follow a geometric Poisson process with unknown intensities, SIAM J. Control Optim., 43, 2004, pp. 1174-1195.

[9] D. G. Luenberger, Optimization by vector space methods. Wiley New York 1969.

[10] H. Nagai and S.Peng, Risk-sensitive dynamic portfolio optimization with partial information on infinite time horizon, Annals of Applied Probability 12, 2002, pp. 173-195.

[11] H. Nagai, and W.J. Runggaldier, PDE approach to utility maximization for market models with hidden Markov factors. To appear in : Progress in Probability (Fifth Seminar on Stochastic Analysis, Random Fields, and Applications), 2006.

[12] B. Oeksendal, The value of information in stochastic control and finance, Australian Economic Papers (Special Issue on Stochastic Models in Economics and Finance), Vol.44 (Dec 2005), pp. 352-364.

[13] H. Pham and P. Tankov, A model of optimal consumption under liquidity risk with random trading times and its coupled system of integrodifferential equations. Preprint 2006.

[14] W.J.Runggaldier, and S. Di Emidio, Computing efficient hedging strategies in discontinuous market models. In : Stochastic Finance (M. do Rosario Grossinho, A.N. Shiryaev, M.L. Esquivel, P.E. Oliveira eds.). Springer Science + Business Media, 2006, pp. 197-212.

[15] W. Schachermayer, Utility Maximization in Incomplete Markets. In: Frittelli, M. and Runggaldier, W. (eds) "Stochastic Methods in Finance". Lecture Notes in Mathematics Vol. 1856. Springer-Verlag, Berlin-Heidelberg, 2004, pp. 255-293.

[16] W. Schachermayer, Optimal investment in incomplete markets when wealth may become negative, Annals of Applied Probability 11, 2001, pp. 694-734. 